\title{
Automatic Homographic Registration of a Pair of Images, with A Contrario Elimination of Outliers
}

\author{
Lionel Moisan ${ }^{1}$, Pierre Moulon ${ }^{2}$, Pascal Monasse ${ }^{3}$ \\ ${ }^{1}$ MAP5, Université Paris Descartes (lionel.moisan@parisdescartes.fr) \\ ${ }^{2}$ IMAGINE/LIGM, Université Paris Est \& Mikros Image (pmo@mikrosimage.eu) \\ ${ }^{3}$ IMAGINE/LIGM, Université Paris Est (monasse@imagine.enpc.fr) \\ Communicated by Frédéric Sur Demo edited by Pascal Monasse
}

\begin{abstract}
The RANSAC [2] algorithm (RANdom SAmple Consensus) is a robust method to estimate parameters of a model fitting the data, in presence of outliers among the data. Its random nature is due only to complexity considerations. It iteratively extracts a random sample out of all data, of minimal size sufficient to estimate the parameters. At each such trial, the number of inliers (data that fits the model within an acceptable error threshold) is counted. In the end, the set of parameters maximizing the number of inliers is accepted.

The variant proposed by Moisan and Stival [7] consists in introducing an a contrario [1] criterion to avoid the hard thresholds for inlier/outlier discrimination. It has three consequences: 1 . The threshold for inlier/outlier discrimination is adaptive, it does not need to be fixed. 2. It gives a decision on the adequacy of the final model: it does not provide a wrong set of parameters if it does not have enough confidence. 3. The procedure to draw a new sample can be amended as soon as one set of parameters is deemed meaningful: the new sample can be drawn among the inliers of this model.

In this particular instantiation, we apply it to the estimation of the homography registering two images of the same scene. The homography is an 8-parameter model arising in two situations when using a pinhole camera: the scene is planar (a painting, a facade, etc.) or the viewpoint location is fixed (pure rotation around the optical center). When the homography is found, it is used to stitch the images in the coordinate frame of the second image and build a panorama. The point correspondences between images are computed by the SIFT [5] algorithm.
\end{abstract}

\section{Source Code}

The source code to reproduce the same results as the demo can be found on the article web page (https://doi.org/10.5201/ipol.2012.mmm-oh). Notice that due to the random component of ORSA, different runs may yield slightly different results. You may also observe slower runs on your machine for large images, as the mosaic construction parts use the multiple cores via OpenMP (http://openmp.org/), and the IPOL server has probably more cores (32 currently).

\section{Supplementary Material}


A first implementation by Moisan and Stival of the method as a Megawave2 (http://megawave. cmla.ens-cachan.fr/) module, called stereomatch.c, can be found in [7].

Possible updates, bug fixes, or enhanced versions of the code can be found at http:// imagine.enpc.fr/ moulonp/AC_Ransac.html. Notice however that such versions are not necessarily peer-reviewed.

Keywords: a contrario method, homography, image matching, robust estimation, RANSAC

\section{Disclaimer}

The present work publishes only the ORSA [7] algorithm as described below (applied to homography estimation). It does not publish the SIFT subroutine which is called by the demo code. SIFT may be updated or replaced by other subroutines.

\section{Background}

\section{$1.1 \quad$ RANSAC}

The RANSAC algorithm is used to estimate parameters of a model fitting some data among which outliers are present, that is part of the data is corrupted. The assumption is that correct data is consensual, while incorrect data is random. Let us note $N_{\text {sample }}$ the number of data samples necessary to estimate the parameters and $n$ the total number of data samples.

1. Randomly draw $N_{\text {sample }}$ random data samples.

2. Estimate parameters Param of the model from these samples.

3. Count number $c$ of inliers among the $n$ data samples given Param, using a threshold $\sigma$ on the residual error.

4. If $c$ is larger than its greatest value up to now, store Param and $c$.

5. Go to step 1 if the allocated number of iterations nIter is not reached.

In point 3 above, distinction between inlier and outlier is done using the parameter $\sigma$, a threshold on the deviation to the model (the "residual error" in regression). Observe that ideally all sets of $N_{\text {sample }}$ data samples should be tested, but this would be too long for even moderate $n$, except maybe when $N_{\text {sample }}=2$. Hence the random selection in step 1 .

As above, we note $N_{\text {sample }}$ the minimal number of data terms to determine a geometric configuration with no remaining degree of freedom. For example:

- $N_{\text {sample }}=1$ for a translation since one correspondence term determines it.

- $N_{\text {sample }}=2$ for a similarity.

- $N_{\text {sample }}=3$ for an affine transform.

- $N_{\text {sample }}=4$ for a homography.

- $N_{\text {sample }}=7$ for a fundamental matrix. 


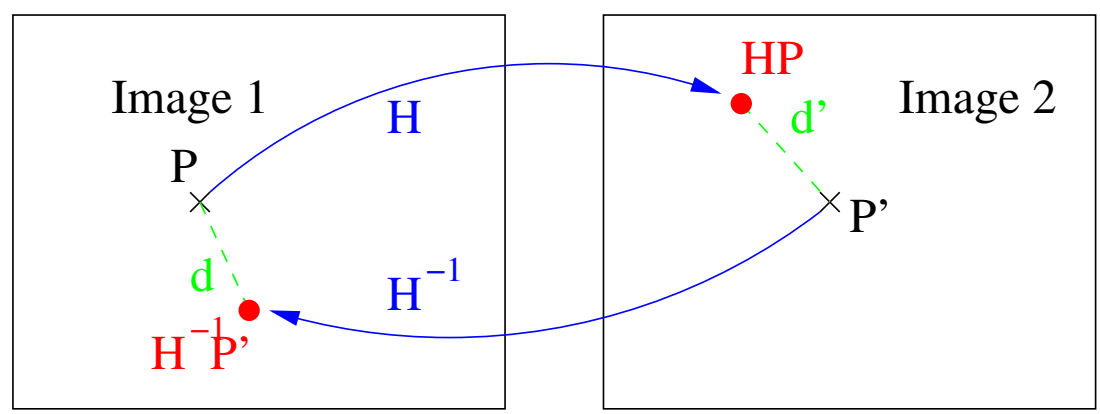

Figure 1: Residual error terms for homography: $d^{\prime}$ (transfer error in second image) or $\max \left(d, d^{\prime}\right)$ (symmetric transfer error)

Of course, in all but the first of these cases, there are degenerate situations that do not permit a unique estimation. The case of fundamental matrix with the 7-point method is interesting in that it can yield up to 3 solutions. We will note $N_{\text {outcomes }}$ the number of possible models estimated by $N_{\text {sample }}$ data terms. In most cases, $N_{\text {outcomes }}=1$.

Line detection example: consider the problem of fitting a line to $n 2$-D points corrupted by noise and outliers. In such a case, two points are required to estimate the parameters of a line $\left(N_{\text {sample }}=2\right)$. A point would be deemed inlier if its distance to the line is less than $\sigma$. Notice that at the end the resulting line is still estimated from only two points, so may not be very precise. Nevertheless at this point we can discard all outliers and compute the regression line of inliers. It is a common procedure to estimate more precisely the model considering only inliers in the end.

Many RANSAC variants differ in their strategy of sampling the data. In any case, there remains the critical parameter $\sigma$. If set too small, many true inliers are ignored because they are classified as outliers. If $\sigma$ is too large, the algorithm allows outliers to mix with inliers and this yields a model of poor precision. The correct choice of this parameter greatly depends on the data and the model considered. The ORSA algorithm finds the right balance between $\sigma$ and number of inliers by controlling the number of false alarms.

\subsection{ORSA}

When evaluating point correspondences between two images, the ORSA (Optimized RANSAC) algorithm uses an a contrario approach to model the expected residual error. Such a background model is based on a uniform distribution of points in the images and random coupling of them. Strong deviations from this background model are considered significant and a natural threshold arises to ensure control of the expected number of false alarms.

As usual in a contrario methods, given a geometric configuration with $k$ inliers among $n$ data terms, its number of false alarms (NFA) is the product of two terms: $\mathrm{NFA}=N_{\text {tests }} P(k, n)$, the number of tests times the probability, which depends on the configuration. From a sample of $N_{\text {sample }}$ correspondences, we compute the model parameters Param. Then the deviation to this estimated model (called residual error, or simply error) is computed for all data, looking for a consensus set. The error term can be the transfer error in second image or the symmetric transfer error. See Fig. 1 for the different error terms in case of homography. All data samples are then sorted by increasing

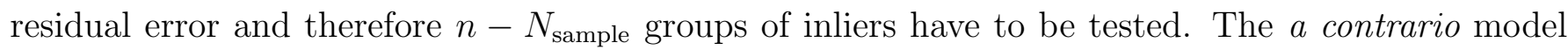
and its NFA measure are here to select the "best" one (the most significant).

Suppose that the inlier/outlier threshold is $\sigma=\epsilon_{k}$, the $k^{\text {th }}$ least error among all $n$ data terms. Given a geometric model, let $\alpha_{0}$ be the probability of a random data term to have error at most 1 (normally expressed in pixels). The probability of error at most $\sigma$ is $p=\sigma^{d} \alpha_{0}$, where $d$ is the dimension ( 1 for distance to a line, 2 for distance to a plane point). 
Let us give the general formula for the NFA, which is a direct generalization of (6) in MoisanStival's article [7] and also mentioned in Rabin et al.'s paper [8]:

$$
\mathrm{NFA}=N_{\text {outcomes }}\left(n-N_{\text {sample }}\right)\left(\begin{array}{l}
n \\
k
\end{array}\right)\left(\begin{array}{c}
k \\
N_{\text {sample }}
\end{array}\right)\left(\epsilon_{k}^{d} \alpha_{0}\right)^{k-N_{\text {sample }}} .
$$

Indeed, if there are $k$ inliers, potentially all $N_{\text {sample }}$ out of them yield the correct configuration. Each one has a probability $\epsilon_{k}^{d} \alpha_{0}$ of being inlier according to the background model (see (2) below for a definition of $\alpha_{0}$ in case of homography). Through the assumption of independence of the data terms, the probability that all $k$ of them are inliers is $\left(\epsilon_{k} \alpha_{0}\right)^{k-N \text { sample }}$ since the $N_{\text {sample ones used }}$ for estimation are automatically inliers (they have error 0 ). The number $k$ of inliers is usually not known in advance, so all values of $k$ are tested (from $N_{\text {sample }}+1$ to $n$ ), which explains the factor $\left(n-N_{\text {sample }}\right)$. So given a sample of $N_{\text {sample }}$ data terms, we compute the geometric model(s). We then collect the errors of all data terms and sort them: $\epsilon_{1}$ to $\epsilon_{n}$. For each possible $k$, we compute the NFA as above, and keep only the least of them, provided it is below some threshold, usually 1.

The parameter $d$ as exponent of $\epsilon_{k}$, representing the dimension of a region of iso-error measure, is 1 in the case of fundamental matrix (length) and 2 for homography (area), see explanation in Section 4.3.

\subsection{Homography}

In this particular demonstration, the ORSA algorithm is tested for homography estimation. A homography registers one image to another taken from pinhole cameras of a rigid scene in any of the two cases:

- the optical center does not move (pure rotation and possibly change of camera settings), or

- the viewed scene is planar.

We have then $N_{\text {sample }}=4$ and $N_{\text {outcomes }}=1$. The geometric error of a correspondence $\left(P_{i}, P_{i}^{\prime}\right)$ is the distance from the point in left image $P_{i}$ mapped to the right image by the homography $H$ to its corresponding point: $\left\|H\left(P_{i}\right)-P_{i}^{\prime}\right\|$. Assuming as background model a uniform distribution of points $P_{i}^{\prime}$, the probability of having error 1 or less is

$$
\alpha_{0}=\pi /\left(w^{\prime} \times h^{\prime}\right)
$$

ratio of areas of a radius 1 disk and of the right image. Finally the specific formula of the NFA is

$$
\mathrm{NFA}=(n-4)\left(\begin{array}{l}
n \\
k
\end{array}\right)\left(\begin{array}{l}
k \\
4
\end{array}\right)\left(\epsilon_{k}^{2} \frac{\pi}{w^{\prime} h^{\prime}}\right)^{k-4} .
$$

To estimate $H$ from 4 correspondences, its coefficients are those of a null vector of an $8 \times 9$ matrix obtained as follows. We write in homogeneous coordinates

$$
\lambda_{i} P_{i}^{\prime}=H P_{i} \quad i=1, \ldots, 4 .
$$

That is,

$$
P_{i}=\left(\begin{array}{lll}
x_{i} & y_{i} & 1
\end{array}\right)^{T}
$$

and $H$ is a $3 \times 3$ matrix. The coordinate vector $\lambda_{i} P_{i}$ represents the same point as $P_{i}$ for any $\lambda_{i} \neq 0$, so that $H$ is defined up to a scale factor. 
These are 3 equations per correspondence, but there is an additional unknown $\lambda_{i}$. We can eliminate it by using the cross product of the two sides:

$$
P_{i}^{\prime} \times\left(H P_{i}\right)=0
$$

which amounts to two independent linear equations on the coefficients of $H$. Since points are not at infinity, $z_{i}^{\prime} \neq 0$, and we can check easily that the first two equations are independent. Writing in the 9 -vector $h$ the coefficients of $H$ in row-major order, we get the system

$$
A_{i} h=0 \quad \text { with } A_{i}=\left(\begin{array}{ccccccccc}
x_{i} & y_{i} & 1 & 0 & 0 & 0 & -x_{i}^{\prime} x_{i} & -x_{i}^{\prime} y_{i} & -x_{i}^{\prime} \\
0 & 0 & 0 & x_{i} & y_{i} & 1 & -y_{i}^{\prime} x_{i} & -y_{i}^{\prime} y_{i} & -y_{i}^{\prime}
\end{array}\right)
$$

Stacking the 4 matrices $A_{1}$ to $A_{4}$ in an $8 \times 9$ matrix $A$, we have to solve $A h=0$, the scale of $h$ being indifferent. This can be found through computation of the SVD of $A$. For technical details, see this Section 4.4 .

\section{Online Demo}

The first time you run the demo on a pair of images, the ORSA algorithm determines automatically the error threshold, which we call precision. In some cases, it can be useful to set manually a maximum error (for example 1 pixel). This can be done through a drop-down menu in the results page. In that situation, the ORSA algorithm still determines automatically the precision, but it is guaranteed to be below the precision set by the user.

The demo offers the possibility to select a rectangle of the first image and try to register only that part. In that case, the SIFT points are computed on the full image but those outside the selection are discarded. Notice that this is not quite the same as applying the SIFT algorithm on the cropped image because of border effects.

The demo uses the symmetric transfer error (see Figure 1), but the code itself has the possibility to use the transfer error in second image.

You can regulate the number of correspondences by modifying the SIFT ratio $s$. The default value is $s=0.6$, which should have very few false positives. Given a SIFT point $P$ of descriptor $\operatorname{desc}(P)$ in image 1 , we match it with $Q$ in image 2 if

$$
Q=\arg \min _{P^{\prime}} d\left(\operatorname{desc}(P), \operatorname{desc}\left(P^{\prime}\right)\right) \text { and } d(\operatorname{desc}(P), \operatorname{desc}(Q))<s \times \min _{P^{\prime} \neq Q} d\left(\operatorname{desc}(P), \operatorname{desc}\left(P^{\prime}\right)\right) .
$$

In other words, $P$ matches the SIFT point of image 2 of closest descriptor, but only if second closest descriptor is at $s$ times this descriptor distance. When $s=1$, the latter condition is always satisfied and all SIFT points in image 1 match with one point in image 2. The number of outliers becomes large with such a high value, typically 60-70\%. To challenge the algorithm even further and have more outliers, we generalize this condition to the following one when $s \geq 1$ :

$$
d(\operatorname{desc}(P), \operatorname{desc}(Q))<s \times \min _{P^{\prime}} d\left(\operatorname{desc}(P), \operatorname{desc}\left(P^{\prime}\right)\right) .
$$

The point $P$ can now match with several points $Q$ in right image. This is a way to add artificially outliers. In our tests on typical examples, we get around $90 \%$ outliers for $s=1.1$.

Here is an example of text output on the Croisette images proposed in the demo page:

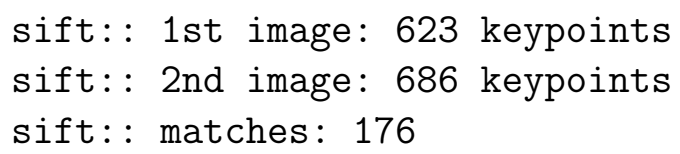




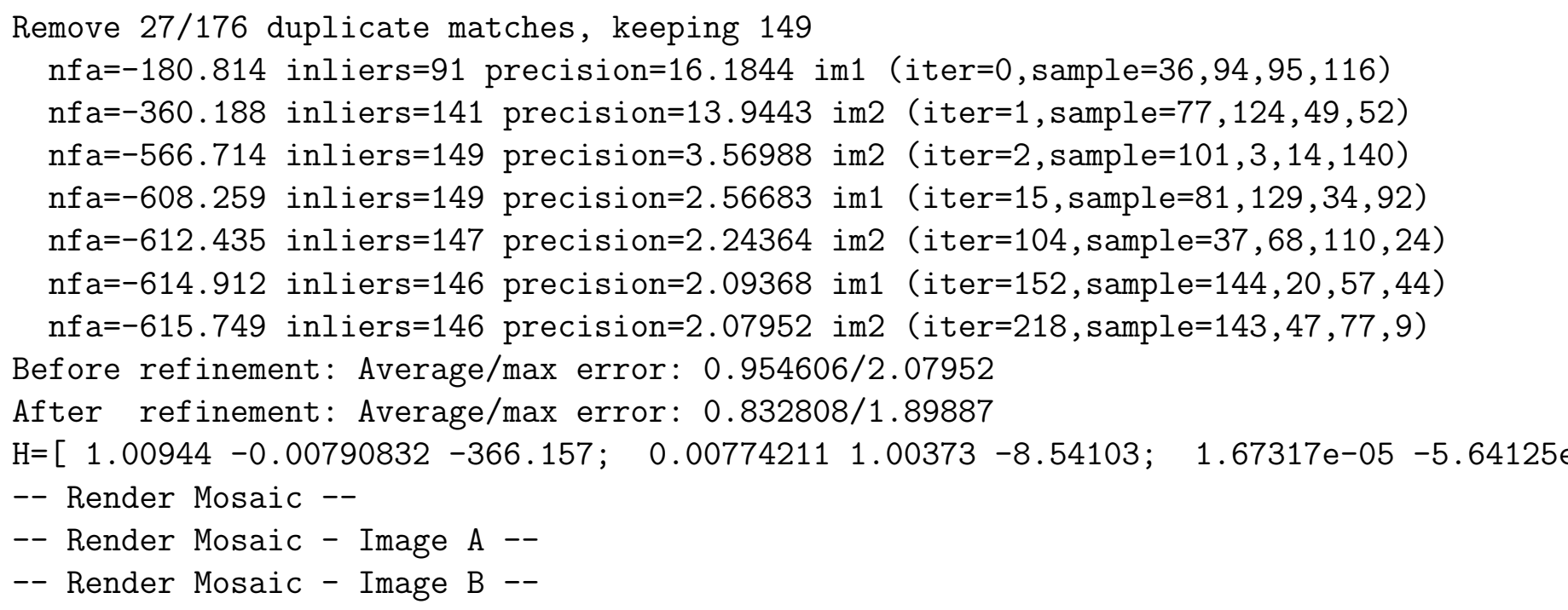

First, results of SIFT are displayed: number of keypoints in each image and number of matches. Some of these matches are exact duplicates (same start and end positions) because SIFT keypoints can be encoded independently with different orientations. The algorithm removes duplicates, here 27. Each time a better model (i.e., lower NFA) is detected, a line is displayed with the base 10 logarithm of the NFA, the number of inliers according to the determined precision (in pixels), the image in which the error is measured, and the iteration number. The indices of the correspondences that were used for model estimation are also displayed. The indices match the line number in the output file of all correspondences (first line being 0). In the end, the RMSE (called "average error" in program's output) and the maximal error (that is, the final precision) after reevaluation of the homography based on all inliers is computed (least square minimization, see Section 4.4 for details). It can be noticed that the final precision (2.30) is slightly higher than the precision of the last iteration (2.25) because of this final least square minimization. The estimated homography $(3 \times 3$ matrix $)$ mapping the first image on the second image is displayed. The homography matrix is normalized so that the last coefficient is 1 .

In the outliers image, the matches are displayed in red and for each outlier a yellow line joining the observed endpoint to the point predicted by the estimated homography is shown. This yellow line can be sometimes hard to distinguish for outliers with small error (but by definition still higher than the precision).

Three mosaic images are produced. The first one is the true mosaic, with the first image warped on the second one and the common part showing both by transparency (average of the original colors). This mosaic has the maximal dimensions of the two images and is rendered such that the middle point of the overlap is centered in the mosaic (see Figure 2). This choice was made to avoid having to build and transfer large images (in case of strong distortion induced by the homography) but to still show at best the precision in the overlap. The other two mosaics are the registered individual images (in the same reference frame as the true mosaic), permitting to check the results by flipping the images.

\section{Algorithm}

\subsection{Generic ORSA Algorithm}

The general ORSA algorithm works as shown in Algo. 1. In the demo, niter=10,000 iterations.

The vector vec_index is the set of indices of data used in the sampling step to compute homography parameters. Initially, it is the identity, but whenever the best meaningful model so far is found 


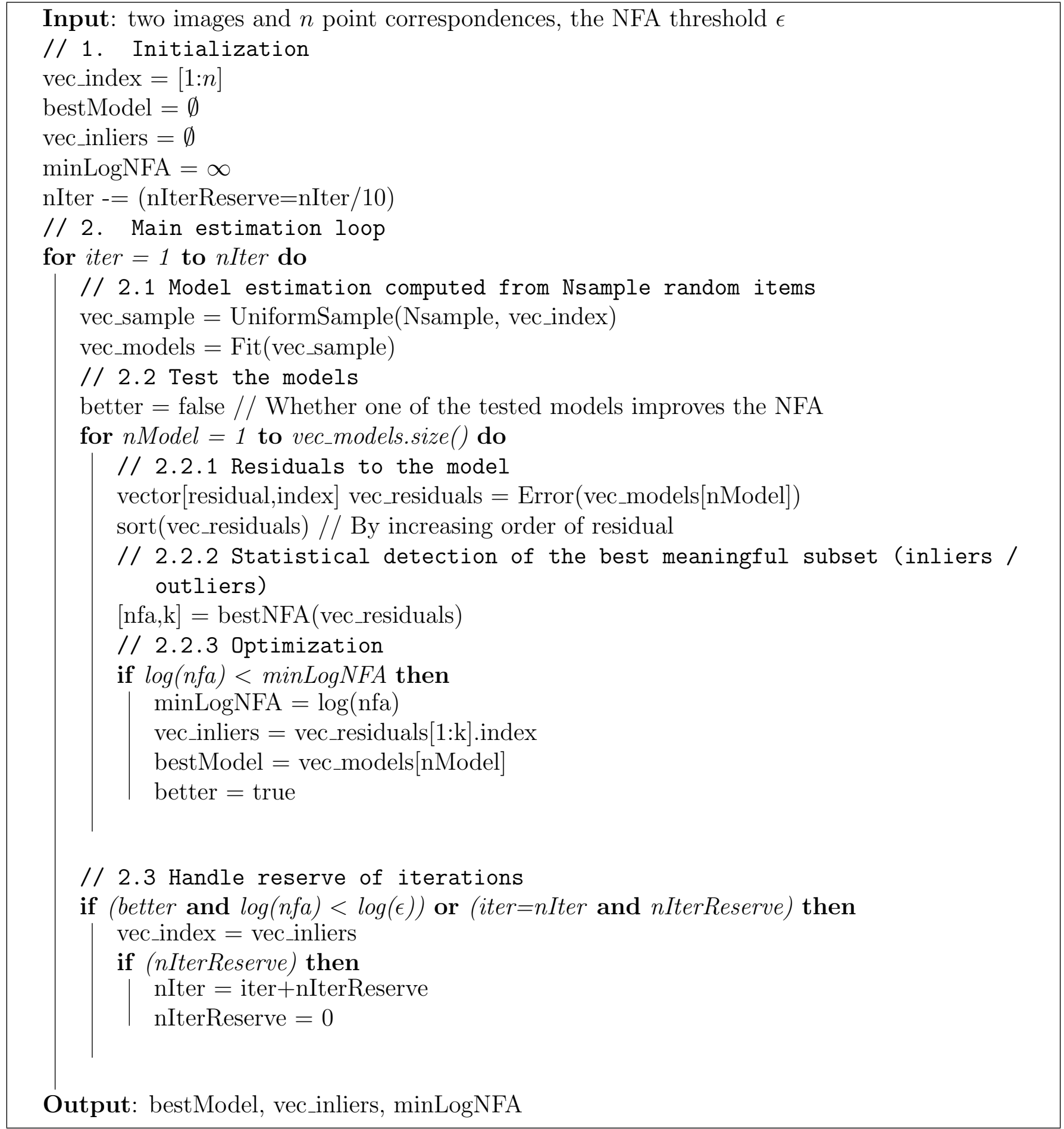

Algorithm 1: ORSA algorithm 


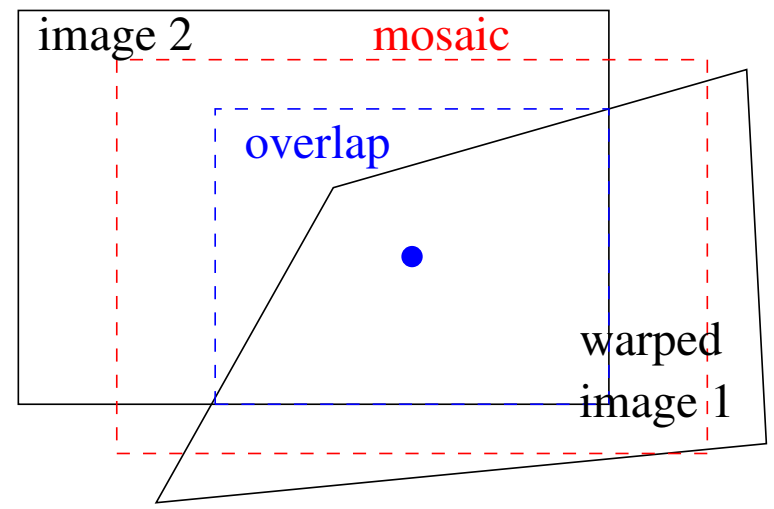

Figure 2: Support of mosaic image. The warped first image is intersected with the second image support. The center of the bounding rectangle of the overlap is fixed as mosaic's center. The mosaic dimension is the maximum of the dimensions of both original images.

(in 2.3), this array is set to inliers, so that next samples will be drawn among inliers. Among them, $N_{\text {sample }}$ are drawn at random and the models are estimated. In the case of homography, there is only one model (possibly 0 for degenerate configurations) so the loop in 2.2 has only one iteration.

In 2.2.1, all residuals $\epsilon_{i}$ are computed and sorted while preserving their index $i$. The rank $k$ yielding the lowest NFA is found by exhaustive search in 2.2.2. If it improves the best NFA so far, inliers' indices are stored in vec_inliers at step 2.2.3, hence the need to keep indices when sorting the residuals, and the model saved.

As in the original implementation [6] by Moisan, $10 \%$ of iterations (nIterReserve) are reserved for optimized sampling in step 1. This reserve is then handled with in 2.3. As soon as a meaningful model is found, subsequent samples will be drawn among its inliers, and only $10 \%$ of iterations will be tested. In presence of few outliers, a meaningful model is found in the first few iterations, so that few more than 1000 iterations $(10 \%$ of nIter $=10,000)$ are tested. However if no meaningful model is found after $90 \%$ of iterations, inliers are still set from the best model found so far and the remaining iterations will draw their samples among them. The idea is that even though no meaningful model has been found, the best found model has a higher probability of containing true inliers. This can help in difficult cases of high outlier ratio.

\subsubsection{Discussion about Parameters: Precision and NFA Threshold $\epsilon$}

Our code seems to depend on two essential parameters: the maximum authorized precision and the NFA threshold $\epsilon$. The precision really depends on the application: in most cases, a precision of a few pixels is the maximum acceptable. Remember that the actual precision is ultimately automatically chosen by ORSA, below the user's input precision.

Concerning $\epsilon$, the NFA threshold, a standard value of 1 is common in a contrario methods. This is also our choice in the demo. Notice however that we are looking here for a unique detection at most, so that accepting a casual registration when comparing two quite different images may look strange. A value such as $10^{-3}$ would seem more reasonable. However, we prefer showing a registration in the demo, however unreliable it may be, instead of the answer "no match". It may however be noted that a value of $\epsilon$ too high may trap ORSA in a local minimum.

\subsection{Homography Check}

Degenerate configurations can occur in homography estimation, such as 3 aligned points among the 4 correspondences. Also the estimated homography can be wild, mapping a square to a self-intersecting 
quadrilateral.

\subsubsection{Wild Homographies}

To reject the wild homographies, we ignore all homographies that are not orientation preserving in the vicinity of the 4 points; in other words, we keep only quasi-affine homographies (see HartleyZisserman [3], Chap. 21), which are the only physically plausible homographies obtained from directly observed endpoints of correspondences (in contrast to, for example, points deduced as intersection of observed lines). For this, we need to ensure that the determinant of the Jacobian $J$ of $H$ is positive. Writing the projective transformation in inhomogeneous coordinates

$$
H(x, y)=\left(\begin{array}{l}
H_{1}(x, y) \\
H_{2}(x, y)
\end{array}\right)=\left(\begin{array}{c}
\frac{h_{11} x+h_{12} y+h_{13}}{D} \\
\frac{h_{21} x+h_{22} y+h_{23}}{D}
\end{array}\right) \text { with } D=h_{31} x+h_{32} y+h_{33},
$$

we compute the Jacobian

$$
J=\frac{1}{D}\left(\begin{array}{ll}
h_{11}-h_{31} H_{1} & h_{12}-h_{32} H_{1} \\
h_{21}-h_{31} H_{2} & h_{22}-h_{32} H_{2}
\end{array}\right)
$$

$J, H_{1}$ and $H_{2}$ being expressed at a same point $(x, y)$. The determinant is then

$$
D^{2} \operatorname{det} J=\left(h_{32} h_{21}-h_{31} h_{22}\right) H_{1}+\left(h_{31} h_{12}-h_{32} h_{11}\right) H_{2}+\left(h_{11} h_{22}-h_{12} h_{21}\right),
$$

and we are interested in the positivity of the right hand side. It is interesting to note that the equation

$$
\left(h_{32} h_{21}-h_{31} h_{22}\right) x^{\prime}+\left(h_{31} h_{12}-h_{32} h_{11}\right) y^{\prime}+\left(h_{11} h_{22}-h_{12} h_{21}\right)=0
$$

represents the image of the line at infinity $z=0$ in the right image (the image of a line $l$ is $H^{-T} l$ and the line at infinity is $l_{\infty}=\left(\begin{array}{lll}0 & 0 & 1\end{array}\right)^{T}$. . More generally, the coefficients involved in the left hand side are those of the last column of the comatrix of $H$, which, divided by $\operatorname{det}(H)$, is the last row of $H^{-1}$. Notice that if the 4 image points are on the positive side of this oriented line, so are points in their convex hull.

Applying this constraint to $H^{-1}$, we get

$$
\left(h_{31} x+h_{32} y+h_{33}\right) / \operatorname{det}(h)>0,
$$

for points $(x, y)$ in the left image. It can be easily checked that if the latter constraint is satisfied, so is the former at corresponding point $\left(x^{\prime}, y^{\prime}\right)$. Therefore, we check the positivity on the 4 left points before accepting the homography as physically plausible.

During computation of the error of the model, each correspondence not satisfying the above constraint gets infinite error, so it cannot be counted as inlier.

\subsubsection{Degenerate Homographies}

Another constraint is that $\operatorname{det}(h) \neq 0$. This is not satisfied typically when 3 of the 4 points are aligned in one image. Numerically, this is difficult to check as the scale of $h$ is arbitrary. On the other hand, we can compute the condition number of $h$, that is the ratio of the extreme singular values. In tests, we observed that a value above 10 never happens for a reasonable homography, so we reject the case. A typical value is rarely above 3 .

Notice that this check is simpler to perform than the collinearity of any triple of points. 


\subsection{Duplicates Removal}

A correspondence is a pair of points $\left(P, P^{\prime}\right)$ with $P$ and $P^{\prime}$ from images 1 and 2 respectively, called the endpoints of the correspondence.

It is essential to remove duplicate correspondences (identical endpoints) before launching ORSA. Typically, the SIFT method can attach multiple orientations, so multiple codes, to keypoints. If a keypoint in one image has several orientations and another keypoint in the other image also has several orientations, the codes of these keypoints can match for these different orientations, and several correspondences with identical endpoints are output. However, we do not discard correspondences sharing only one endpoint: although they are mutually exclusive, we cannot know in advance which is the correct one, if any.

Such a configuration is a clear violation of the background model and can yield dramatic results. Suppose that A and B are two correspondences with identical endpoints. It can happen that $\mathrm{A}$ is drawn to participate to a trial sample with 3 other correspondences. If $\mathrm{B}$ is among the other 3 , the estimated homography gets rejected because of its high condition number. However, if B is not among them, B fits perfectly with the estimated homography (error $=0$ or tiny), since A does. This 5 -correspondence configuration gets a very $\operatorname{low} \log (\mathrm{nfa})$, possibly $-\infty$, and is deemed the best.

\section{Implementation}

You can download the source code from the article web page (https://doi.org/10.5201/ipol. 2012. mmm-oh), tested with $\mathrm{g}++4.6$ under Linux and with Microsoft Visual C++ 2010 under Windows. The main code is under license LGPLv3, with some supporting parts under simplified BSD license.

\subsection{Generic ORSA Algorithm}

The ORSA algorithm is implemented as a method of an abstract class 'OrsaModel', which calls essentially two virtual functions that must be implemented by subclasses:

- Fit (indices, vec_models), which outputs the model(s) computed by the indexed correspondences.

- Error (model, index), which returns the square residual error of the indexed correspondence according to the input model.

The method Fit returns usually a unique model, but the algorithm accepts several possible models or possibly none. The maximum number of models estimated by a sample of correspondences is indicated by the virtual function $\operatorname{NbModels}()$ and is used in the NFA computation $\left(N_{\text {outcomes }}\right)$. The method Error returns a square residual error: the square root extraction is replaced by taking half of the logarithm, which has to be computed in the NFA.

Aside from NbModels(), two other virtual methods need to be implemented by subclasses: SizeSample() returns the size of a sample $\left(N_{\text {sample }}\right.$ in NFA formula) and DistToPoint() indicates whether the error distance is from a point to a point (such as in case of homography) or from a point to a line (such as for fundamental matrix). This information is necessary to compute the probability of a point correspondence to be within a given precision.

A protected parameter logalpha0 is the logarithm of $\alpha_{0}$, the probability of having an error at most 1 . This must be initialized by each subclass in its constructor (all logarithms are base 10). 


\subsection{Normalized Coordinates}

It is important (even crucial for fundamental matrix [4]) to estimate the models from points with normalized pixel coordinates to avoid badly conditioned linear systems. As these points are used over and over, the class OrsaModel normalizes the points input to its constructor by matrices $\mathrm{N} 1$ and N2 respectively, encoding zoom-translation according to image dimensions $\left(w_{i}, h_{i}\right)$ :

$$
N_{i}=\left(\begin{array}{ccc}
1 / s_{i} & 0 & -w_{i} /\left(2 s_{i}\right) \\
0 & 1 / s_{i} & -h_{i} /\left(2 s_{i}\right) \\
0 & 0 & 1
\end{array}\right) \text { with } s_{i}=\sqrt{w_{i} h_{i}} .
$$

The normalized points are stored in protected members $\mathrm{x} 1$ and $\mathrm{x} 2$ of OrsaModel $(2 \times n$ matrices $)$, so as $\mathrm{N} 1$ and N2, so that derived classes have access to them. Correspondences are designed by their index, that is the column number in $\mathrm{x} 1$ and $\mathrm{x} 2$ in methods Fit and Error.

As the model is estimated according to normalized points, the normalization has to be taken out at the end. The virtual method ComputeModel (indices, model) returns the model estimated from all inliers (designed by indices) in original coordinates, that is without normalization. As the way to "de-normalize" the model is specific to each model, the actual code is left as implementation of a virtual method Unnormalize(model) in each subclass.

\subsection{NFA Computation}

The terms in the NFA formula may include large binomial coefficients, so logarithms of all terms are considered instead. Since they have to be computed for various $k$, the binomial coefficients are tabulated in logc_n (varying $k$ among $n$ point correspondences) and $\log c_{\_} \mathrm{k}\left(N_{\text {sample }}\right.$ among varying $k$, up to $k=n)$. We can rewrite the NFA formula in terms of logarithm:

$$
\log \mathrm{NFA}=\log N_{\text {outcomes }}\left(n-N_{\text {sample }}\right)+\log \left(\begin{array}{l}
n \\
k
\end{array}\right)+\log \left(\begin{array}{c}
k \\
N_{\text {sample }}
\end{array}\right)+\left(d \log \epsilon_{k}+\log \alpha_{0}\right) \times\left(k-N_{\text {sample }}\right) .
$$

The fixed term $N_{\text {outcomes }} \times\left(n-N_{\text {sample }}\right)$ involved in NFA has its log computed once as 'loge0'. The actual NFA computation takes place in private method OrsaModel : : bestNFA:

$$
\begin{aligned}
& \text { logalpha }=\text { logalpha0 }+ \text { multError } * \log 10(\mathrm{e}[\mathrm{k}-1] . \text { first }) ; \\
& {[\ldots] \text { loge } 0+\text { logalpha } *(\text { double })(\mathrm{k}-\operatorname{startIndex})+\log c_{-} \mathrm{n}[\mathrm{k}]+\log \mathrm{c}_{-} \mathrm{k}[\mathrm{k}] \text { [..] }}
\end{aligned}
$$

This is inside a loop with varying $\mathrm{k}$ (number of inliers), as in NFA formula. The term logalpha is the log of the probability of having an error sqrt (e[k-1].first): remember that e[k-1].first is the square error $(k-1$ is the last among $k$ inliers as numeration starts at 0$)$. Its log is multiplied by multError $(d=2 \times$ multError $)$, which is 1 or 0.5 depending on whether the error is distance to a point (homography) or to a line (fundamental matrix), indicated by method DistToPoint(). Indeed, in the first case the probability varies as the square of the error (area of a disk), while in the second case it varies linearly as the error, so we have to take square root of error, thus multiplying the $\log$ by 0.5 .

The probability logalpha is multiplied by k-startIndex, which is $k-N_{\text {sample }}$. The binomial terms are added, so as the constant term loge 0.

\subsection{Homography Model Estimation}

The class HomographyModel is derived from OrsaModel. $N_{\text {sample }}=4, N_{\text {outcomes }}=1$, DistToPoint() returns true. Error is measured in image 2. The term logalpha0 is the (log of the) ratio of the areas of a disk of radius 1 and of the image, that is $\pi /\left(w_{2} \times h_{2}\right)$. Since we will be working in normalized coordinates, in which distances are multiplied by $\left(w_{2} \times h_{2}\right)^{1 / 2}$, we have: 


$$
\text { logalphaO_ }=\log 10\left(\mathrm{M}_{-} \mathrm{PI} /(\mathrm{w} 2 *(\text { double }) \mathrm{h} 2) /\left(\mathrm{N} 2 \_(0,0) * \mathrm{~N} 2_{-}(0,0)\right)\right) \text {; }
$$

since $\mathrm{N} 2(0,0)$ is the scale factor.

In finding the nullspace of the matrix $A$, we compute its SVD (actual computation is done by code extracted from ccmath (http://freecode.com/projects/ccmath) library). To avoid degenerate situations (in which 3 of the 4 points are aligned), we check the lowest two singular values of $A, \sigma_{n}$ and $\operatorname{sigma}_{n-1}$. If the lowest, $\sigma_{n}$, is less than $10^{-2}$ times the highest singular value $\sigma_{1}$, we consider that it corresponds to a null vector. If the second lowest, $\sigma_{n-1}$, also satisfies this condition, we consider the solution as not unique unless $\sigma_{n}<0.1 \sigma_{n-1}$, and we reject the estimation.

In RANSAC algorithm, it is customary to recompute the model based on inliers by least square error at the end. We minimize the RMSE (Root Mean Square Error) by the same method, finding the lowest singular value of matrix $A$, built from inliers only. It can be noticed that we have two different measures for quality of a homography: the NFA and the RMSE. The ORSA algorithm is based on NFA and the refinement step on RMSE. We can wonder whether the new model after refinement, though optimal according to RMSE, also makes the NFA decrease. To test that, we devised an iterative procedure as follows:

1. Find inliers through ORSA algorithm, compute NFA.

2. Refine the model by minimization of RMSE based on inliers.

3. Sort data based on refined model by increasing residual error.

4. Recompute the rank $k$ yielding lowest NFA.

5. If the optimal set of inliers has changed go to 2 .

This option can be turned on by the method OrsaModel : setRefineUntilConvergence. Notice however that there is no guarantee of convergence of this procedure. In our tests, almost all pairs of images converge in a couple of iterations. We could not observe a significant difference in the results. This option is not proposed in the demo, but can be tested by running the code.

\section{$5 \quad$ Examples}

Figure 3 is an example of pose estimation of a DVD cover in a scene. Notice that all 4 corners of the cover happen to match at the same corner in the right image. The error between the detected outlier endpoint and the true position predicted by the estimated homography is shown in yellow. Among outliers, part of the $\mathrm{Y}$ is matched to the A, a real error, while a point near the dog's head matches at a correct but imprecise location (the inlier/outlier threshold is automatically determined at 2 pixels). In the mosaic image, the most important errors are located on the actor's name, as the blur witnesses, because few correspondences are located there.

In the next example, Figure 4, almost all but one correspondences are considered inliers, the remaining one being slightly less precise. Notice that in the mosaic image, the ghost of the bus front, present only in the right image, is visible.

\subsection{Influence of Precision Parameter}

The following images show the influence of the precision parameter on the results. With no constraints, we get almost all correspondences as inliers, with a final precision of 2.2 pixels. With a precision of 1 pixel, we get about 40 correspondences rejected as outliers in various parts of the images, while with a precision of 0.5 pixel, only 65 inliers are detected. They are concentrated on the lower half of the image, because the motion does not obey precisely a homography model. 

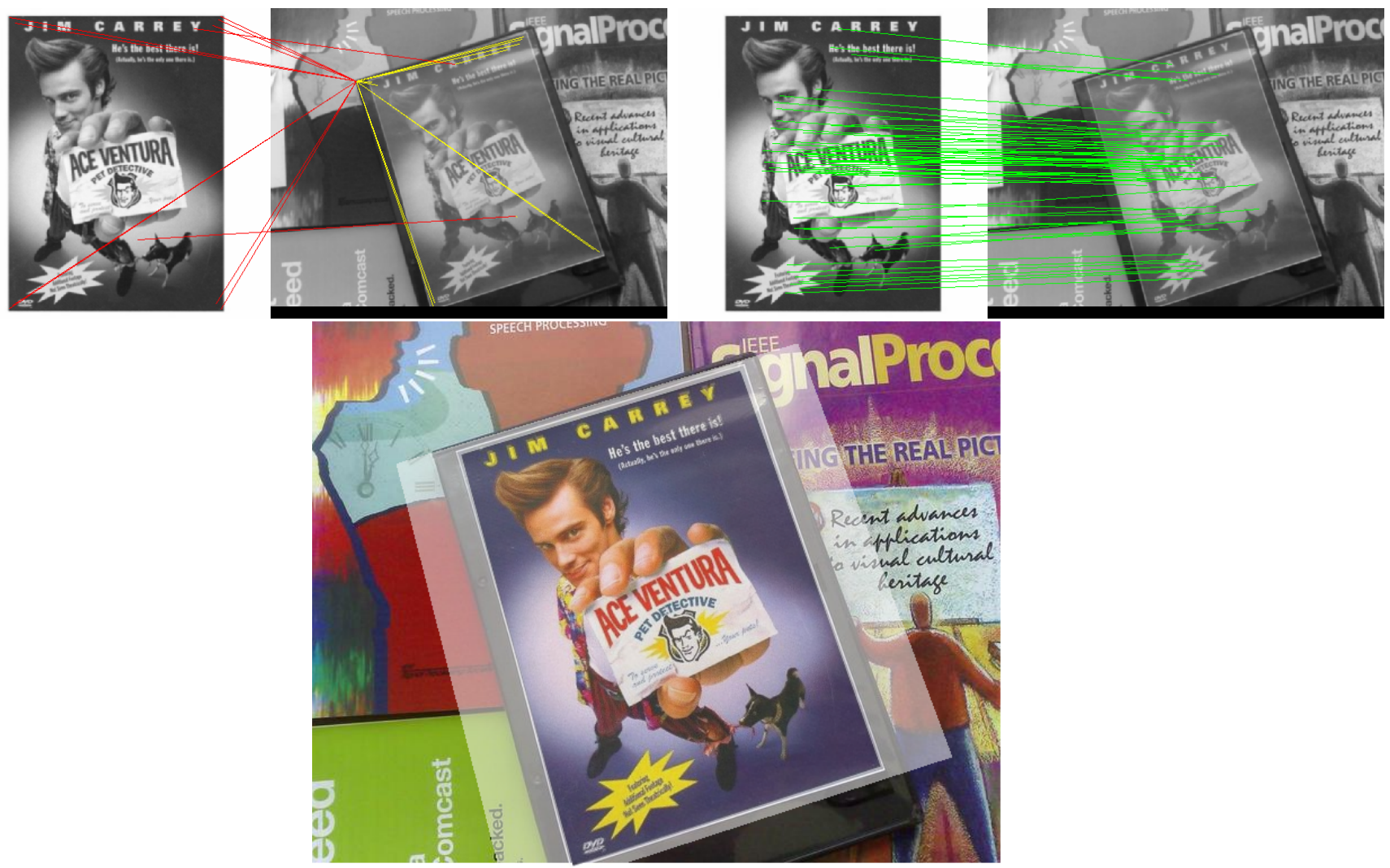

Figure 3: DVD cover experiment. Outliers (top-left) and inliers (top-right), mosaic (bottom)
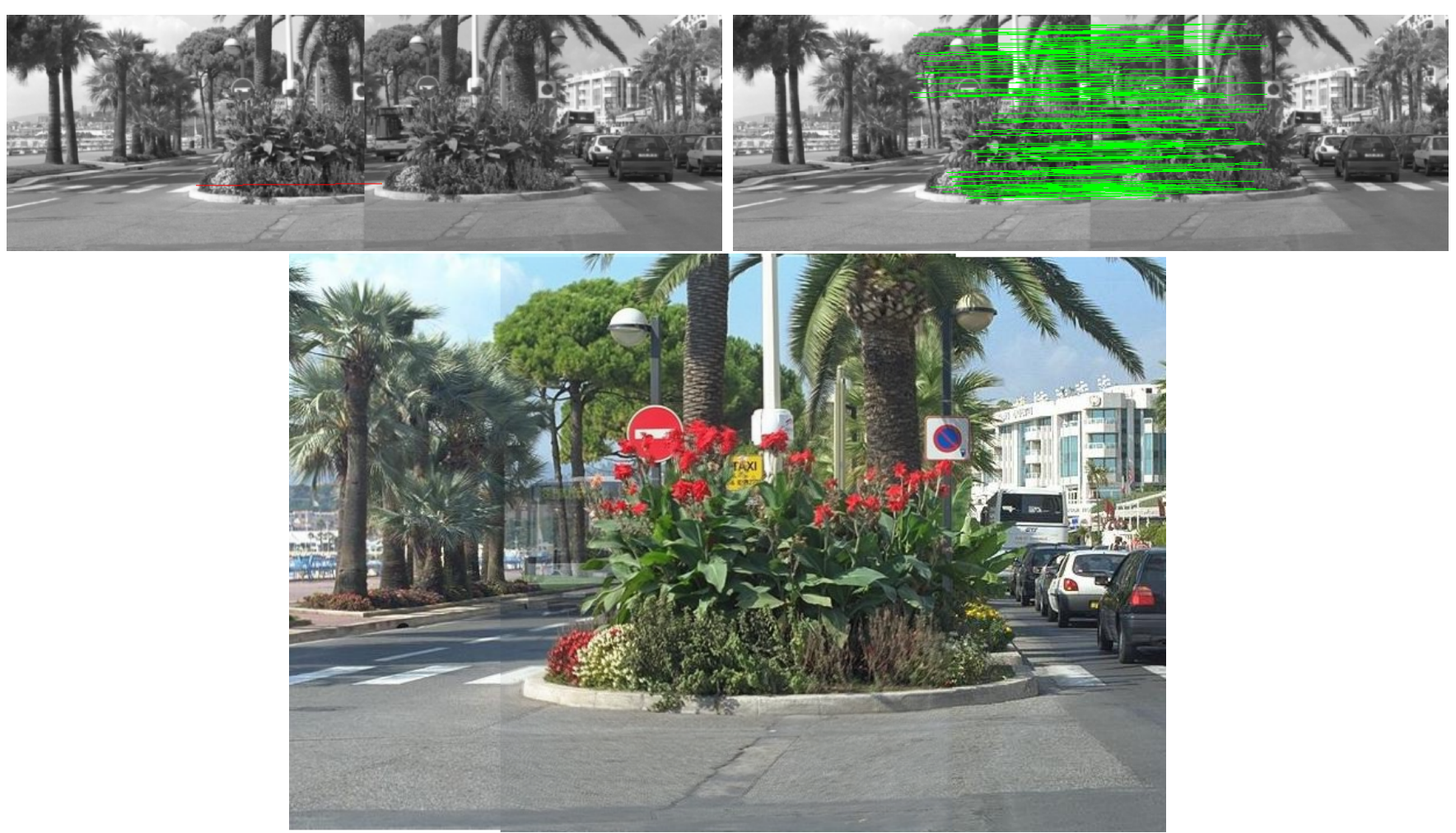

Figure 4: Croisette images experiment. Outliers (top-left) and inliers (top-right), mosaic (bottom) 


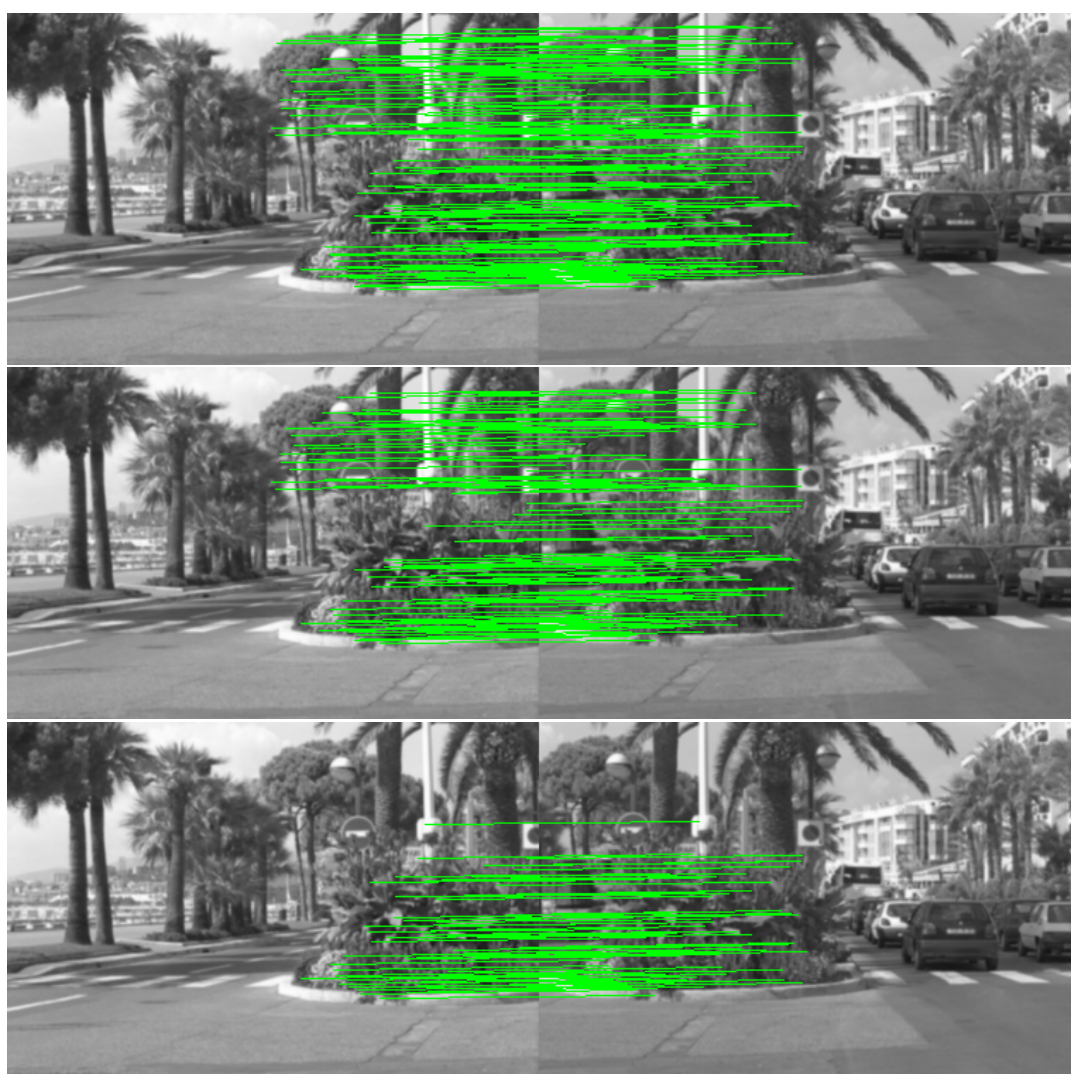

Figure 5: Croisette inliers with different precisions: precision=automatic (top), precision=1 (middle), precision $=0.5$ (bottom).

\subsection{High Outlier Ratio}

To demonstrate the resilience of the algorithm to high outlier ratio, we perform the following experiment: we crop a part of the right image in the Croisette pair above. The overlap between the images is less than $20 \%$ of the left image. We set the SIFT ratio $s=1$, that is accept for each SIFT point in left image its best matching SIFT point in right image. We get 609 correspondences, 43 being deemed inliers at the end, thus an estimated outlier ratio of $93 \%$. Here is an extract of the program output:

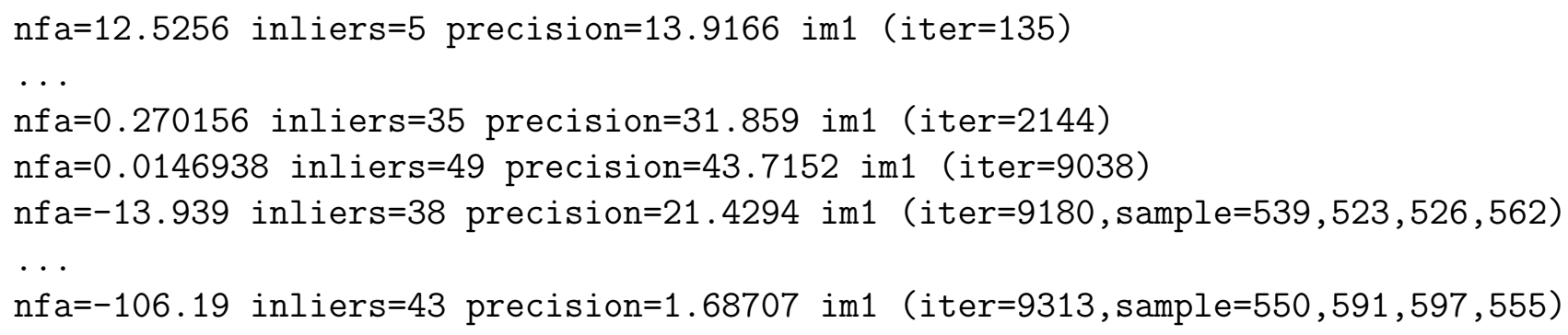

At iteration 2144, the algorithm has not found a meaningful model yet. At iteration 9000 (90\% of total iterations), it still applies optimized sampling, drawing samples among the 35 inliers of the best model so far. The first improvement at iteration 9038 is not yet meaningful, but at iteration 9180 the first meaningful homography is detected, though with a large precision of 21 pixels. The final model is found with a precision of less than 2 pixels. This example shows the interest of optimized sampling even before a meaningful model is detected. The inliers and outliers are shown in Fig. highout. 

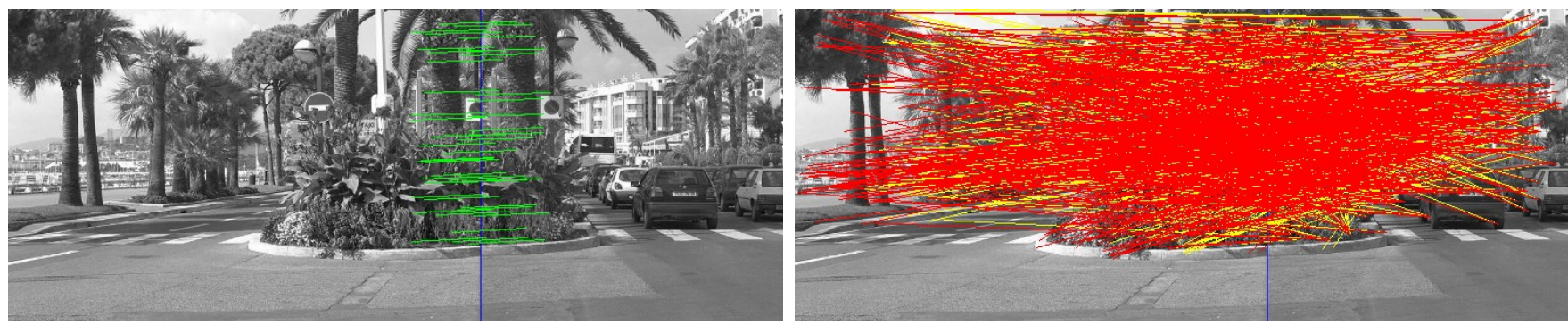

Figure 6: Registration of a crop of left Croisette image with right image, SIFT ratio $s=1$. Inliers $(43 / 609=7 \%)$ and outliers $(566 / 609=93 \%)$

\subsection{Failure Cases}

The only failure cases we met were for images with very few matches. First if there are only 4 correspondences, there is no NFA associated, a homography could still be estimated, but we have no means to judge its quality. With only 5 correspondences, the only possibility is to take all as inliers. In that case, we have NFA $=5 p$, with $p$ the probability associated to the error of the $5^{\text {th }}$ point. If the image is large and this error moderate, we can still get a meaningful homography.

This is what happens in the following example: correspondences 1 to 4 are correct, while 5 is wrong. Even though its error is large (280 pixels) the associated probability is still moderate, as the image is 3.5 megapixels:

$$
\pi \times 280^{2} /\left(3.5 \times 10^{6}\right)=0.07<1 / 5
$$

The main reason is that correspondences are restricted to a small section of the right image (compared to its full size). The mosaic shows that the resulting homography is wild, but errors of the 5 control points are not huge, 70 pixels on average with a maximum of 90 pixels (after refinement based on the 5 correspondences). Of course, such large errors would be unacceptable in a real application and fixing the precision parameter at some reasonable value (5 pixels for example, to be fairly tolerant) would prevent such detection.

A more problematic case with a few more correspondences is illustrated below:

Among these 9 correspondences, 5 are actually correct $(1,2,5,6,8)$. However the match is not very precise (4.5 pixels after optimization but up to 9 pixels for point 6 when the homography is estimated from the 4 other points), probably because the sheet is not quite flat. The NFA has a log of -0.5 , barely meaningful, even though this yields a correct homography:

Note also that 4 points in the left image $(4,5,7,9)$ match to the same point in right image (only 5 is correct). Unfortunately, the above registration is not the best achievable NFA. The latter has a value of -2.38 (in $\log$ ) with points $(2,3,7,8)$ and a precision of 92 pixels! This is obtained using points $(4,5,9)$ as inliers and rejecting 1 and 6 , which are actually correct. However, after least square minimization, the error becomes huge: several thousands of pixels, obviously because points $4,5,7$, and 9 are supposed to be mapped to the same point. A former version of the code did not check the condition of the homography matrix, and so did not reject it.

Notice that such an error would not have happened if an actual combination of 4 among the 5 true correspondences were found before: this would have constrained the set of inliers, and the incorrect above configuration would never have been tested as further samples would be among true inliers. The outcome depends on chance. The RANSAC algorithm aims at minimizing the NFA, but there is no guarantee to reach the global minimum. In fact, the optimization step of ORSA speed-up converges in practice to a local minimum by constraining the set of samples. As in the first example, setting a reasonable maximal precision beforehand rejects the wrong estimation. 


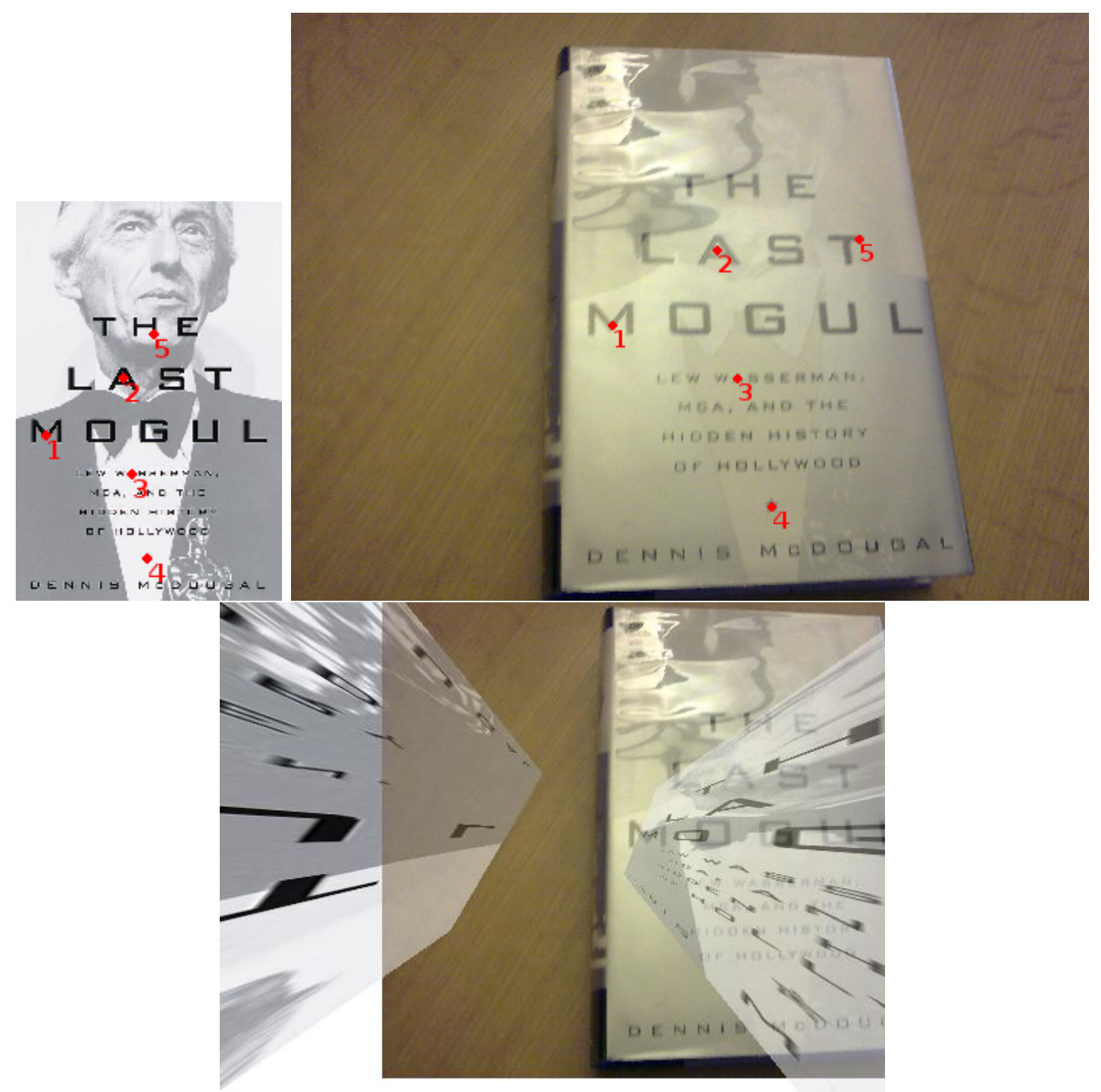

Figure 7: Mosaic of Mogul images 

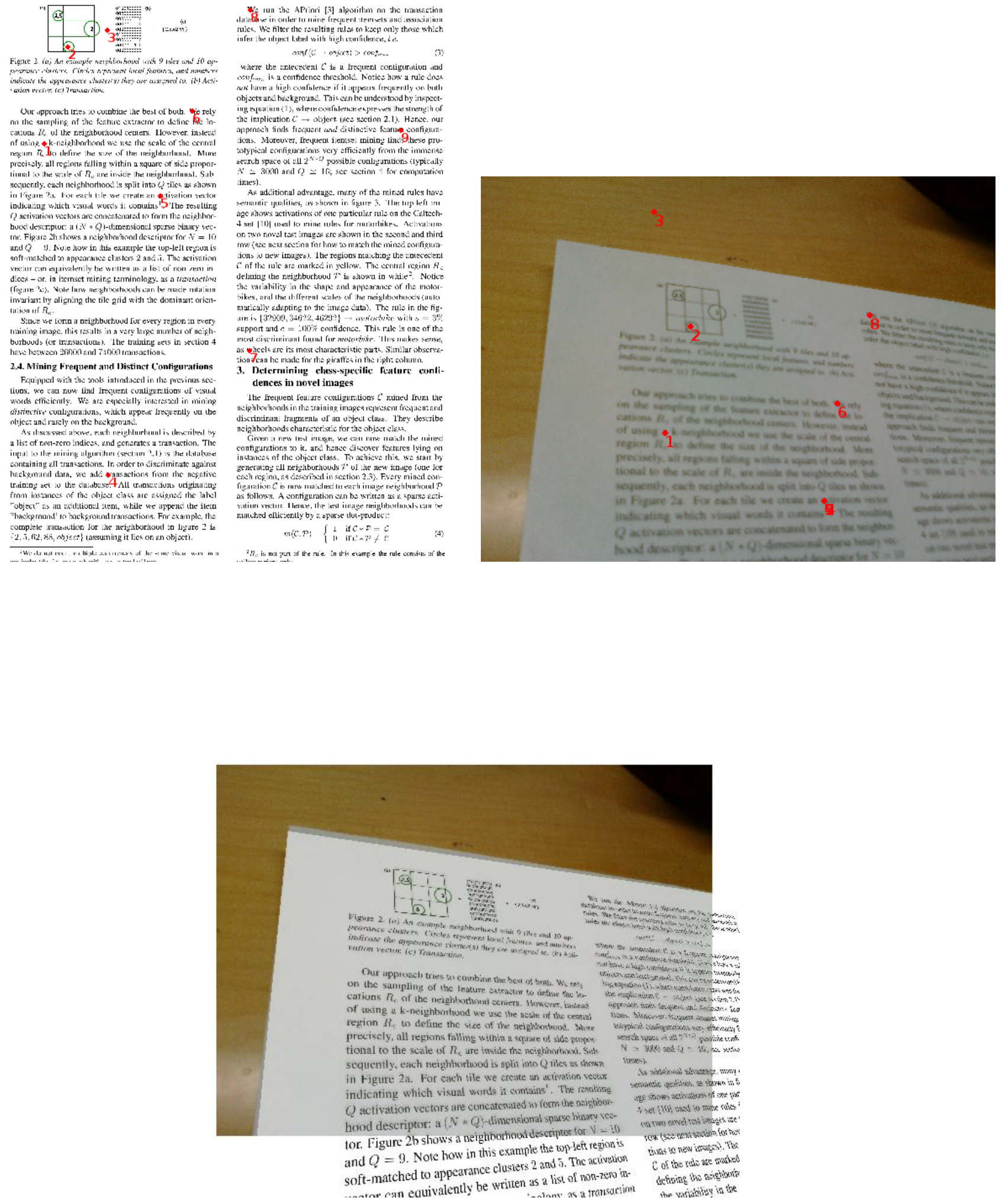

Figure 8: The correct mosaic found when constraining precision;10 


\section{Acknowledgments}

This work was supported by Centre National d'Études Spatiales (CNES) through the MISS project and by Agence Nationale de la Recherche grant ANR-09-CORD-003 (Callisto project). The authors would like to thank two reviewers, among them Viorica Patraucean, for their in-depth reading, testing, and interesting discussion of this article. Jean-Michel Morel's judicious advice and testing are gratefully acknowledged.

\section{Image Credits}

(3.). mvs.html) by V.R. Chandrasekhar, D.M. Chen et al.

Mogul images, Stanford mobile visual search database (http://www. stanford.edu/ dmchen/mvs.html) by V.R. Chandrasekhar, D.M. Chen et al.

Print images, Stanford mobile visual search database (http://www.stanford.edu/ dmchen/mvs.html) by V.R. Chandrasekhar, D.M. Chen et al. all other images (C) Pascal Monasse, CC-BY

\section{References}

[1] A. Desolneux, L. Moisan, and J.M. Morel. Meaningful alignments. International Journal of Computer Vision, 40(1):7-23, 2000. http://dx.doi.org/10.1023/A:1026593302236.

[2] M.A. Fischler and R.C. Bolles. Random sample consensus: a paradigm for model fitting with applications to image analysis and automated cartography. Communications of the ACM, 24(6):381395, 1981. http://dx.doi.org/10.1145/358669.358692.

[3] R. Hartley and A. Zisserman. Multiple view geometry in computer vision. Cambridge Univ Press, 2nd edition edition, 2004. ISBN 978-0521540513.

[4] R.I. Hartley. In defense of the eight-point algorithm. Pattern Analysis and Machine Intelligence, IEEE Transactions on, 19(6):580-593, 1997. http://dx.doi.org/10.1109/34.601246.

[5] D.G. Lowe. Distinctive image features from scale-invariant keypoints. International journal of computer vision, 60(2):91-110, 2004. http://dx.doi.org/10.1023/B:VISI.0000029664. 99615.94.

[6] L. Moisan. Source code stereomatch.c. http://www.math-info.univ-paris5.fr/ moisan/ epipolar/download.php?file=stereomatch.c, 2007.

[7] L. Moisan and B. Stival. A probabilistic criterion to detect rigid point matches between two images and estimate the fundamental matrix. International Journal of Computer Vision, 57(3):201-218, 2004. http://dx.doi.org/10.1023/B:VISI.0000013094.38752.54.

[8] J. Rabin, J. Delon, Y. Gousseau, and L. Moisan. Mac-ransac: a robust algorithm for the recognition of multiple objects. In Fifth International Symposium on 3D Data Processing, Visualization and Transmission (3DPVT 2010), Paris: France, 2010. 